\title{
ARTICULOS
}

CRITICA, Revista Hispanoamericana de Filosofta

Vol. XXIV, No. 71 (agosto 1992): 3-33

\section{REALISMO EPISTEMOLÓGICO, REFERENCIA Y VEROSIMILITUD}

Alejandro Cassini CONICET Universidad de Buenos Aires

El tema del realismo ha ocupado una posición cada vez más destacada en la filosofía de la ciencia de los últimos años. Se han renovado los argumentos en favor del realismo científico, a la vez que han proliferado las posiciones antirrealistas. Una de las dificultades más recurrentes en este debate es la ambigüedad del término realismo, heredada de una larga y compleja tradición filos6fica. El uso descuidado o arbitrario de esta palabra frecuentemente ha oscurecido las discusiones. Como consecuencia de ello, no ha resultado fácil determinar con precisión qué implica una concepción realista del conocimiento científico. ${ }^{1}$ En este trabajo no me ocuparé de argumentar en favor o en contra del realismo. Abordare, en cambio, la tarea previa de esclarecer el concepto de realismo epistemológico, es decir, la posición realista respecto de las teorías científicas. Ante todo, distinguiré dos especies fundamentales de realismo: el realismo ontológico, o realismo acerca de las entidades que componen el mundo, y el realismo epistemológico, o realismo acerca de las teorías científicas. Luego, señalaré las relacio-

1 Cfr. Boyd, 1989. 
nes entre realismo, verdad y referencia, y argumentaré que una epistemología realista se halla comprometida tanto con la idea de verdad (más precisamente, de verosimilitud) de los enunciados de una teoría, como con la de referencia de los términos, en especial los teóricos, de dicha teoría. Sostendré que la existencia de descripciones verdaderas es necesaria para determinar la referencia de los enunciados de una teoría, y para asegurar la identidad del referente de dos o más teorías diferentes (particularmente, pero no exclusivamente, cuando se produce un cambio de teoría). Ejemplificaré esta tesis analizando la manera de hacer referencia en las teorías de partículas elementales. Concluiré que el realismo referencial, o cualquier otro intento de elaborar una teoría realista del conocimiento científico prescindiendo del concepto de verdad, resulta insostenible.

\section{Realismo ontológico y realismo epistemológico}

Es conveniente distinguir dos sentidos básicos del término realismo cuando se emplea en contextos filosóficos:

a) El realismo ontológico es la tesis que afirma que el mundo existe por sí mismo, con independencia del conocimiento o la conciencia que se tenga de él. En particular, el mundo es independiente de la percepción, el pensamiento, el lenguaje o cualquier otra actividad mental de los sujetos humanos (o, eventualmente, de las máquinas o de los sujetos no humanos). La existencia de las entidades que componen el mundo no es afectada por la acción de la mente y, en consecuencia, estas entidades pueden existir aunque no haya mente alguna que las perciba. El realismo ontológico es esencialmente una posición acerca del modo de existencia de las cosas. Esta clase de realismo se opone tradicionalmente al idealismo, es decir, a la tesis general de que el mundo no existe independientemente de la conciencia o de la mente (o, en todo caso, de alguna mente privilegiada). 
En el ámbito del mundo físico, el realismo ontológico sostiene que los sistemas físicos poseen determinadas propiedades (por ejemplo, posición en el espacio-tiempo, momento, energía, etc.) por sí mismos, con independencia de que estas propiedades sean conocidas, observadas, medidas o registradas por los sujetos humanos o los instrumentos empleados por ellos. En particular, las propiedades de los sistemas físicos nunca son producidas o creadas por los procesos de conocimiento, observación y medición, sino que existen previamente, preexisten, a tales procesos. Así, la medición de la masa de un objeto físico presupone que este objeto posee una masa determinada antes de que se efectúe tal medición. La medición registra simplemente la masa preexistente de ese objeto, más allá de errores experimentales o perturbaciones previsibles o calculables. Lo mismo vale para las demás propiedades físicas. Incluso en la mecánica cuántica, donde hay conocidas y serias dificultades sobre este punto, una solución realista del problema de la medición debería ajustarse en principio a estas características generales. $^{2}$

2 No prejuzgaré aquí si esta solución es posible, pero es indudable que será sumamente diff́cil (cfr., en general, Rae, 1986). Históricamente, se han alterado las interpretaciones realistas y antirrealistas de la mecánica cuántica, siendo esta última la más extendida (cfr. Brush, 1980, que proporciona una buena síntesis). Posteriormente, la refutación experimental de las desigualdades de Bell ha conducido al abandono de las teorías de variables ocultas locales y a la discusión en términos de no separabilidad y no localidad de los sistemas cuánticos (sobre este punto, el tema de moda desde hace años, la bibliografía es enorme; cfr., entre otros, Mermin, 1981; D'Espagnat, 1981; Ortoli-Pharabod, 1984; Deligeorges, comp., 1984; Selleri, 1986; Teller, 1986; Redhead, 1989; Healey, 1991). De allí se han extraído frecuentemente interpretaciones antirrealistas. Sin embargo, el uso de "realismo" suele ser poco claro en estas discusiones. En principio, ni la acción a distancia, si existiera, ni la idea de que las entidades microfísicas son no separables cuestionan el realismo ontológico. Por otra parte, el realista en mecánica cuántica no se halla constreñido a sostener que los sistemas cuánticos son como partículas clásicas (como afirma Gibbins, 1987, IX y 14, en un libro por lo demás excelente); sino que puede admitir que sean entidades enteramente diferentes de las partículas. 
El realismo ontológico en general contiene como una de sus especies al realismo platonizante (lo llamamos así a falta de un mejor nombre), es decir, a la afirmación de que ciertas entidades de carácter no físico, tales como clases, números, relaciones, significados y proposiciones, tradicionalmente llamadas universales, y en la actualidad a veces entidades abstractas, existen por sí mismas y deben considerarse como parte del inventario del mundo. Como se advierte, el realismo platonizante es una tesis acerca de la existencia autónoma de determinadas entidades, por lo cual, resulta una especie del realismo ontológico. Tradicionalmente, el realismo platonizante se opone al nominalismo, es decir, a la posición que considera que los universales no son entidades sustanciales que existan por sí mismas con independencia de la mente o del lenguaje.

b) El realismo epistemológico (en adelante abreviado como $\mathrm{RE}$ ) es la tesis muy general de que es posible conocer el mundo tal como éste es en sí mismo. Más precisamente, nuestro conocimiento alcanza las cosas en sí, y las describe y explica en su propia naturaleza, tal como son. Por esta razón, las teorías, que son el mejor producto de nuestro conocimiento, son susceptibles de ser verdaderas o verosímiles (esto es, parcial o aproximadamente verdaderas). El RE sostiene al respecto que la verdad o falsedad de las teorías científicas no depende de los sujetos de conocimiento, ni del consenso, ni de la autoridad, ni de ningún factor social, psicológico o epistémico. La verdad de las teorías depende exclusivamente del mundo mismo. Son verdaderas las teorías que describen y explican, de una manera aproximada, el mundo tal como es en sí mismo. Se advierte por esta formulación que el RE se halla comprometido con alguna forma de teoría de la verdad como correspondencia. Para el RE la existencia de cualquier entidad que forma parte del mundo es independiente de las teorías que se refieren a tal entidad. Las teorías científicas, si son verosímiles, siempre hacen referencia a entidades que preexisten a las teorías mis- 
mas. Las teorías no crean, ni constituyen, ni determinan sus objetos o las propiedades de éstos. Únicamente los idealizan, describiéndolos de una manera parcial, es decir, no dan cuenta de todas sus propiedades, sino de algunas que se consideran relevantes. Según el $\mathrm{RE}$, podemos conocer la existencia tanto de cualquier entidad que forme parte del universo (sea ésta observable o no), como la de cualquiera de sus propiedades. Esto no implica que sea posible conocer de manera exhaustiva y completa todas las entidades que existen y todas sus propiedades. Pero sí implica que no hay entidades que sean incognoscibles por principio. Todas pueden ser objeto de descubrimiento. El RE admite que la descripción y explicación científicas son capaces de progresar indefinidamente, aunque en general no lleguen a ser completas.

El RE aplicado a las teorías acerca del mundo físico sostiene que es posible conocer las propiedades que posee un sistema físico tal como éstas son en sí mismas. En particular, las propiedades de los sistemas físicos no son creadas ni modificadas de un modo incontrolable por los procesos de conocimiento, observación y medición realizados por los sujetos humanos o sus instrumentos. Ante todo, los actos puramente mentales, como el de pensar, no pueden ejercer ninguna acción sobre los objetos físicos del mundo exterior. En el caso de la medición, por el contrario, se produce una interacción física entre el instrumento de medición y el objeto medido y, consiguientemente, esta interacción entraña un intercambio de energía entre ambos. Esta interacción, a diferencia de los procesos de conocimiento, es capaz de modificar las propiedades del objeto medido. Lo mismo sucede al iluminar un objeto microfísico mediante radiación electromagnética con el fin de observarlo; cuando la energía de las ondas incidentes es comparable con la de la partícula observada, las propiedades de ésta, por ejemplo el momento, resultan alteradas. Desde el punto de vista del RE, se deberá considerar en tal caso que la perturbación introducida por las mediciones no es un obstáculo insalvable y 
que, o bien es despreciable por su pequeñez, o bien es calculable y predecible. Cuando esto no es posible, como ocurre en la mecánica cuántica, se debe considerar que si los resultados de las mediciones de ciertas propiedades son indeterminados es porque las propiedades mismas de los objetos microfísicos son indeterminadas, al menos, de manera simultánea. El RE no implica, en efecto, que todas las propiedades reales sean precisamente determinadas y puedan expresarse por medio de un único número real. ${ }^{3}$ Lo único que el RE afirma es que las propiedades de un sistema físico nunca dependen del observador ni de la teoría.

El RE se opone en general al fenomenismo, es decir, a la tesis de que conocemos las cosas tal como se nos aparecen, como fenómenos, y no tal como son en sí mismas. El modo en que se nos aparecen las cosas depende de nuestro sistema cognoscitivo, de nuestra percepción, de nuestro lenguaje o de nuestras teorías; en síntesis, de nuestra mente en sentido amplio. Sólo conocemos fenómenos, y éstos son algo relativo a la mente y diferentes de las cosas en sí mismas. En el ámbito específico de las teorías científicas, el RE se opone al instrumentalismo, según el cual las teorías no son verdaderas o falsas ni describen las cosas en sí, sino que sólo poseen adecuación empírica, proporcionan un modo de explicar y predecir los fenómenos, sin pretender ofrecer una descripción verdadera de la realidad. El instrumentalismo debe considerarse como una especie de fenomenismo, puesto que limita el alcance de las teorías científicas a los fenómenos.

Las relaciones que existen entre el RE y el realismo ontológico se pueden establecer de la siguiente manera. En primer lugar, el RE implica el realismo ontológico, pues sostener

3 Así, por ejemplo, la desigualdad de Heisenberg, $\Delta p \cdot \Delta q \geq h / 4 \pi$, puede interpretarse de manera que signifique que las entidades cuánticas no poseen simultáneamente posición y momento determinados (cfr. Teller, 1979). Esta interpretación realista, como se sabe, no es la única posible ni la más popular. 
que es posible conocer las cosas tal como son en sí mismas presupone que éstas tienen una existencia autónoma. Si no la tuvieran, no podrían ser conocidas de la manera en que son en sí, sino tal como se presentaran a un sujeto, o relativamente a una determinada teoría, es decir, como fenómeno. En segundo lugar, el realismo ontológico no implica el RE, ya que la tesis de la existencia por sí de las cosas nada dice acerca del conocimiento de éstas ni sobre las teorías que podamos elaborar sobre ellas. Es admisible sostener que las cosas existen por sí mismas, pero también que no es posible conocerlas tal como son en realidad. Nuestro conocimiento podría no alcanzar las cosas en sí mismas. La combinación del dogmatismo realista en materia de ontología con el fenomenismo o incluso con el escepticismo epistemológico quizá no sea muy frecuente, pero al menos resulta posible. En síntesis, el RE presupone el realismo ontológico, pero no a la inversa, ya que éste es independiente del RE.

Esta caracterización del realismo, aunque somera y general, permite aislar el RE y buscar sus implicaciones. Un examen de algunos trabajos recientes sobre el tema del realismo muestra que ello no siempre se consigue, y que no es raro hallar confusiones entre el RE y alguna de sus especies. ${ }^{4}$ La relación entre los conceptos de realismo y verdad es aquí decisiva, puesto que es la que mayores problemas ha generado. Tal como lo hemos presentado, el realismo ontológico es independiente de la idea de verdad, en tanto que es una tesis acerca del modo de existencia de las cosas. El RE, en cambio, implica el concepto de verdad, pues trata acerca de nuestro conocimiento del mundo y de la correspondencia que existe entre este conocimiento y la realidad. Nuestra posición entraña el rechazo de dos caracterizaciones del realismo que adoptan posiciones extremas.

4 Cfr. Harré, 1986; Fine, 1986; Hooker, 1987; Boyd, 1989 y 1990; y Jones, 1991. No sugiero, por supuesto, que todo lo que estos autores afirman sea erróneo. 
Ante todo, si se sostiene que el realismo es básicamente una tesis semántica, caracterizada por la aceptación del principio de bivalencia y el resto de la semántica clásica, ${ }^{5}$ se excluye con ello el realismo ontológico y se pasan por alto los problemas metafísicos propios de la oposición entre realismo e idealismo. Por otra parte, cuando se afirma que el realismo es sólo una tesis ontológica, la que sostiene que la realidad existe independientemente del sujeto, la mente o el conocimiento, ${ }^{6}$ se limita sin razón el concepto genérico de realismo a una de sus especies y, de ese modo, se deja de lado el problema del conocimiento y el de la relación de las teorías con la realidad. La distinción que hemos realizado entre las dos especies de realismo evita el carácter unilateral de estas dos posiciones, a la vez que recoge lo mejor de ambas parte. Además, tiene la ventaja, a diferencia de otras distinciones, ${ }^{7}$ de recoger buena parte de los usos del término realismo a lo largo de la historia de la filosofía.

\section{Verdad y referencia}

Consideramos ahora el concepto de RE con mayor detalle. Según creo, una epistemología realista se halla comprometida con los conceptos de verdad y referencia, de una manera tal que no puede prescindir de ellos. Más precisamente, el RE está comprometido con: a) la verdad parcial, verosimilitud o aproximación a la verdad de los enunciados o sistemas de enunciados que componen las teorías científicas, y b) el carácter referencial de al menos algunos términos teóricos importantes de cada teoría.

5 Ésta es, en lo esencial, la posición de M. Dummett (cfr. Dummett, 1982, y la crítica de Gryling, 1992).

6 Es la posición de Devitt (1984) y Grayling (1992), aunque este último llama "epistemológico" al problema de la existencia de una realidad independiente de la mente. Por su parte, defienden la tesis de que el realismo implica verdad, con argumentos diferentes de los nuestros, Taylor (1987) y Newton-Smith (1989).

7 Como la de Horwich, 1982, o las de Hellman, 1983. 
El realismo tradicional, desde Aristóteles, consideró siempre que las teorías científicas estaban integradas por enunciados verdaderos en sentido simple y absoluto. Sin embargo, el $\mathrm{RE}$ resulta difícilmente defendible en el contexto de esta concepción tradicional de la verdad. En la actualidad, tanto por razones teóricas como históricas, se ha renunciado al concepto de verdad absoluta de las teorías. Se ha logrado un consenso aceptable en considerar que toda teoría científica es susceptible de ser corregida, modificada o directamente abandonada. La historia de las ciencias proporciona buenos argumentos para creer que el conocimiento científico es siempre provisorio. La ciencia se concibe, entonces, como una actividad de búsqueda sin final, en la cual la dinámica del cambio de teorías nunca se detiene. En consecuencia, no puede admitirse que la verdad absoluta sea una propiedad de las teorías científicas, puesto que la verdad absoluta no es modificable ni revocable. En el realismo contemporáneo el concepto tradicional de verdad absoluta ha sido reemplazado por el de verdad parcial o verosimilitud, el cual, en un sentido muy amplio, implica la idea de una aproximación indefinida a la verdad. El RE implica, entonces, que las teorías científicas son verosímiles; que su contenido de verdad o grado de verosimilitud puede compararse; y que, en principio al menos, es posible que las teorías científicas se aproximen indefinidamente a la verdad. Esta idea de aproximación a la verdad no entraña la de convergencia, es decir, la de la conservación de todo el contenido de verdad de las teorías anteriores por parte de las teorías posteriores. ${ }^{8} \mathrm{El} \mathrm{RE}$ puede admitir la pérdida parcial de ciertos contenidos veritativos en el curso de un cambio de teoría.

La tarea de ofrecer una definición precisa del concepto de verosimilitud, así como los criterios para la comparación del grado de verosimilitud de diferentes teorías, presenta problemas técnicos serios y complejos. Aún no se ha desarrollado una

${ }^{8}$ Cfr. Devitt, 1984, pp. 113-117; Boyd, 1990; Schwartz, 1990. 
teoría de la verdad parcial que suscite consenso. Un problema persistente es el de los portadores de verosimilitud. La verdad absoluta, en el sentido de la teoría de la correspondencia del realismo tradicional, es una propiedad de los enunciados más simples, mientras que la verdad de los enunciados compuestos, los sistemas de enunciados o las teorías se define con base en la verdad de los enunciados simples. En cambio, en las teorías de la verdad parcial aún no está del todo claro si la verosimilitud es una propiedad de los enunciados aislados, de ciertos sistemas de enunciados o de una teoría en su totalidad. Esto sucede, en parte, porque las teorías tradicionales de la verdad, como la de la correspondencia, están basadas en el supuesto de que la verdad es abseluta y, por tanto, no se pueden trasladar fácilmente al ámbito de la verdad parcial. Con todo, es posible señalar que una teoría de la verosimilitud aceptable debería sostener que los portadores de verdad parcial son los enunciados simples, pues sólo de esa manera se ofrecería una alternativa global frente a las teorías de la verdad absoluta. De lo contrario, cuando se considera que únicamente las teorías en su totalidad son verosímiles y se define la verosimilitud de una teoría apelando, de una manera $u$ otra, a la verdad o falsedad de los enunciados que la componen, no se desprende uno de la verdad absoluta tradicional, ya que ésta sigue aplicándose a los enunciados de la teoría. ${ }^{9} \mathrm{El}$ RE está, sin embargo, estrechamente ligado a la teoría de la verdad como correspondencia, en tanto éste afirma que los enunciados de una teoría se refieren al mundo real y que la verdad o falsedad de tales enunciados depende exclusivamente del estado del mundo. Conciliar la verdad parcial de los enunciados con la idea de correspondencia con la realidad es un problema fundamental que debe resolver una teoría de la verosimilitud acorde con el RE.

9 Así ocurre, por ejemplo, en la teorf́a de la verosimilitud propuesta por Newton-Smith, 1981, cap. 8. 
El segundo compromiso del RE sostiene que los términos de las teorías científicas son referenciales o descriptivos. Ello vale especialmente para los términos de mayor grado de teoricidad, los cuales, desde el punto de vista realista, nunca pueden considerarse como totalmente carentes de referencia, como simples instrumentos o ficciones útiles para la explicación y predicción de los fenómenos. Apelando a una terminología tradicional, diremos que para el realista los términos que significan entidades o sustancias se refieren a cosas realmente existentes en el mundo, a entidades extralingüísticas y extrateóricas. Por su parte, los términos que significan atributos o propiedades de esas entidades se refieren a propiedades reales de ellas.

El realista se halla compelido a postular la existencia de tantas entidades y propiedades como sea necesario para que una teoría dada sea verosímil. Este compromiso no implica que todos los términos de la teoría sean genuinamente referenciales. Cuál sea la cuota de términos referenciales que requiere una teoría para poseer un grado determinado de verosimilitud es algo difícil de precisar. Depende de las complejas relaciones existentes entre los conceptos de referencia y de verdad parcial. De un modo general, resulta plausible establecer lo siguiente: si la teoría fuera verdadera en sentido absoluto, todas las entidades y propiedades especificadas por la teoría deberían postularse como existentes, y, por tanto todos los términos de la teoría serían referenciales. Puesto que se admite que la teoría es solamente verosímil, es posible aceptar que algunos términos de la teoría no se refieren a entidades ni a propiedades reales. No obstante, es indispensable que haya algunos términos teóricos. fundamentales o importantes que sean genuinamente referenciales. No es razonable que una teoría que carezca en absoluto de términos referenciales sea considerada como verosímil. Así, por ejemplo, no consideraríamos como verosímil una teoría de partículas elementales en la cual el término genérico "partícula" y sus especies ("quarks", "lep- 
tones", etc.) no sean referenciales. Sin embargo, podríamos aceptar que algunos términos de esa teoría (por ejemplo, algunos discutidos en la actualidad, como "partículas virtuales", "polarización del vacío" o "apantallamiento de la carga de color") no tengan referencia genuina. Si falta la referencia a todos los términos teóricos de una teoría, ésta no puede tener correspondencia con la realidad y, por tanto, tampoco es susceptible de ser verdadera o de poseer un cierto grado de verosimilitud. Tal teoría podría considerarse útil o empíricamente adecuada, pero no verosímil. También es plausible suponer que cuanto mayor sea el grado de verosimilitud de una teoría, mayor será el número de términos referenciales que contenga y menor el número de los no referenciales. Sería interesante obtener mayores precisiones cuantitativas sobre esta hipótesis, pero ello es una tarea ardua y no constituye el objetivo de este trabajo.

\section{Realismo sin verdad}

Según he argumentado hasta aquí, el RE no puede prescindir de los conceptos de referencia y verdad, los cuales, desde la perspectiva realista, resultan inseparables. Contra este punto de vista se ha sostenido que es posible distinguir el realismo de la verdad del realismo referencial. ${ }^{10} \mathrm{De}$ acuerdo con esta posición, el realismo referencial puede mantenerse con independencia del realismo de la verdad. El realismo referencial afirma que los términos centrales de una teoría científica denotan entidades que existen por sí mismas, con independencia de la teoría en cuestión o de cualquier otra. Este tipo de realismo, según declaran sus partidarios, no necesita suponer que los enunciados de la teoría son verdaderos, ni que esa teoría ofrece una descripción verdadera de las entidades a las que se refiere. El supuesto básico que permite hacer esta separación entre referencia y verdad es la tesis de que es posible efectuar

10 R. Harré es el propulsor de esta posición (cfr. Harre, 1986, p. 65 y ss., véanse también pp. 97-99). 
actos de referencia exitosos mediante el empleo de descripciones falsas. De esta manera, sería posible asegurar la referencia de una teoría sin necesidad de utilizar ni presuponer enunciados verdaderos.

Se argumenta que, una vez desligado del concepto de verdad, el realismo referencial permite dar cuenta de la refutación de teorías y del proceso dinámico que implica el reemplazo de una teoría por otra. Más precisamente, permite admitir el cambio de teoría sin perder el referente, de manera tal que sea posible afirmar que dos o más teorías, de las cuales una está vigente y las otras han sido rechazadas, se refieren a la mis$m a$ clase de entidades. Así, supongamos que $T_{1}$ y $T_{2}$ son dos teorías diferentes de la física de partículas elementales, que tienen en común, entre otros, el término "electrón". Los enunciados de $T_{1}$ acerca de los electrones podrían ser todos falsos y, además, $T_{1}$ podría ser reemplazada por otra teoría $T_{2}$ que se considerara superior a $T_{1}$ de acuerdo con determinados criterios. No obstante, aun en ese caso, $T_{1}$ sería una teoría acerca de los electrones, de la misma manera que $T_{2}$. La referencia de los términos de una teoría, según esta posición, se puede mantener aunque ésta se considere totalmente falsa. Eso significa que la referencia de los términos de una teoría es independiente de la verdad o falsedad de los enunciados que la componen.

La posición del realismo referencial, tal como ha sido expuesta en los dos párrafos anteriores, me parece insostenible. Y ello por dos razones básicas: en primer lugar porque, según creo, no existe la posibilidad de efectuar una referencia exitosa sin el empleo de alguna descripción verdadera; en segundo lugar, porque una teoría no puede preservar la referencia de otra teoría si considera falsos a todos los enunciados de aquélla. Sostendré, en contra del realismo referencial, que los conceptos de referencia y verdad no son separables para el realista. Consiguientemente, argumentaré que: a) la realización de un acto de referencia exitoso implica el uso de alguna descripción verdadera del objeto referido, y que b) la conservación de la 
referencia en el pasaje de una teoría a otra implica la conservación de algunos enunciados verdaderos de la primera teoría, los cuales también deben considerarse verdaderos en la segunda.

Analicemos primero el problema de las descripciones falsas. Uno de los supuestos del realismo referencial es que éstas no son obstáculo para alcanzar una referencia exitosa. Podemos, por ejemplo, señalar a lo lejos y decirle a otra persona "mira aquel auto negro". Dicha persona identifica el auto al cual queremos referirnos, aunque reconoce que no es negro, sino azul. Parecería, entonces, que hemos logrado efectuar una referencia exitosa mediante el empleo de una descripción falsa. Pero esto es ilusorio. En tales casos la referencia sólo es posible si va acompañada de actos ostensivos. El éxito de actos de referencia como el de nuestro ejemplo se debe en gran parte al acto de ostensión y en nada al uso de la descripción falsa. Si falta el acto de ostensión, la referencia mediante una descripción falsa generalmente fracasa. Podemos comprobarlo con ejemplos de la percepción cotidiana, como el que indicamos antes. Si le decimos por teléfono a alguien "mira el auto negro que está a tu izquierda", con seguridad esa persona no logrará identificar el auto al cual pretendemos referirnos, si ocurre que dicho auto no es negro y/o no se halla a su izquierda. Incluso en el caso de que el contexto sea favorable, por ejemplo, si hay un único auto en el campo visual, probablemente el acto de referir falle. Si resultara efectivo, el éxito se debería al hecho de que la descripción empleada contiene elementos verdaderos, en este caso, intenta referirse a un supuesto auto negro como un auto. Si tratáramos de referirnos a ese auto diciéndole "mira aquel paraguas verde", es decir mediante una descripción que no contiene elemento verdadero alguno, tal descripción falsa no sólo no proporcionaría una referencia exitosa, sino que desconcertaría totalmente al oyente. En consecuencia, podemos establecer con bastante certeza que no es posible efectuar un acto de referencia exitoso mediante el empleo de descripciones 
totalmente falsas y en ausencia de la posibilidad de realizar actos de ostensión.

La importancia de los actos de ostensión ha sido exagerada, generalmente como producto de la adhesión a una concepción empirista del conocimiento y del lenguaje. Se advierte, sin embargo, que en ámbitos tales como el de la física teórica la referencia ostensiva con frecuencia es difícil o directamente imposible. ${ }^{11}$ Por ejemplo, en la física de partículas elementales no hay posibilidad de percibir directamente $y$, por tanto, tampoco de señalar las entidades a las que se refieren las teorías de partículas. No podemos indicar que allí hay un electrón o un quark. Sólo conocemos tales partículas por medio de ciertos efectos macroscópicos que éstas producen. A menudo esos efectos son el extremo de una larga cadena causal en la que se procede a amplificar los efectos microscópicos, tales como los producidos por la desintegración de una partícula. ${ }^{12}$

Si se quiere hacer referencia a una partícula tal como un electrón, se necesita disponer de algún enunciado verdadero que lo describa. Este enunciado no necesariamente tiene que expresar una propiedad esencial o definitoria de los electrones. Puede simplemente enunciar alguna propiedad causal de esta clase de entidades, por ejemplo, la de producir un cierto brillo en un tubo de rayos catódicos. Es cierto que se podría señalar el brillo en el tubo de rayos catódicos y decir "mira esa corriente de electrones". Pero, en verdad, no se hace con ello un acto de ostensión directa de los electrones; sólo se indica un efecto macroscópico causado por estas partículas. Lo mismo sucede cuando se observan las fotografías de estas partículas en una cámara de burbujas. Para que la indicación de estos

11 Consecuentemente, los cientificos y filósofos de tendencia empirista han adoptado una posición instrumentalista frente a las teorías de la física fundamental (cfr. Van Fraassen, 1980, y también Laudan, 1984).

12 Cfr. Cohen-Tannoudji y Spiro, 1990, pp. 252-293, para una descripción de los diferentes métodos de observación de partículas. Más adelante analizamos varios casos. 
efectos constituya un acto de referencia genuina a los electrones, se debe presuponer la verdad de ciertos enunciados, tales como "el electrón causa cierto brillo en un tubo de rayos catódicos", o "el electrón produce determinada huella en la cámara de burbujas en ciertas condiciones". Estos enunciados no son otra cosa que descripciones verdaderas del electrón. ${ }^{13}$ Sin estas descripciones la referencia no puede llevarse a cabo. Si el electrón no causa realmente un brillo en un tubo de rayos catódicos y la descripción anterior es falsa, entonces, al señalar el brillo en el tubo no hemos hecho referencia a electrones. Podemos hacer ostensión de ciertos efectos macroscópicos de los electrones, pero para que ello sea un auténtico acto de referencia necesitamos emplear alguna descripción que implique alguna propiedad del electrón. Pero si la referencia ha de ser exitosa, la descripción tiene que ser verdadera y, consecuentemente, el electrón debe poseer la propiedad en cuestion. Si todas nuestras descripciones del electrón fueran falsas, no podríamos identificar un cierto tipo de entidad como un electrón. En consecuencia, no tendríamos modo de hacer referencia a tal entidad.

\section{Identificación de partículas elementales}

Consideremos más de cerca los problemas que plantea la identificación de las partículas en la física de partículas elementales y analicemos algunos casos específicos. En general, la identificación de una partícula elemental o subatómica presupone la aceptación de una amplia porción de conocimientos de la física teórica, que va mucho más allá de una simple descripción verdadera. Si tomamos una fotografía de ciertas huellas en

13 Se advertirá la semejanza entre estas descripciones y las tradicionales reglas de correspondencia para los términos téricos, pero no siempre las descripciones que se empleen en los actos de referencia serán de este tipo. 
una cámara de burbujas o en una cámara de chispas y decimos, por ejemplo, “aquí se desintegró un pión" o "aquí se produjo un par electrón-positrón", estamos haciendo una interpretación de ciertos indicios sensibles con base en determinada teoría física. No observamos directamente los sucesos microfísicos, sino sus efectos, convenientemente registrados y amplificados (en general las imágenes finales son reconstruidas y analizadas por computadoras). Para considerar que señalando una fotografía hemos hecho referencia a entidades tales como partículas elementales, debemos suponer que una parte relevante de la física de partículas es verdadera. Podemos prescindir de la teoría de los detectores de partículas, los amplificadores y todo el instrumental de observación y registro. Se puede conceder que toda esta teoría no es importante para la determinación de la referencia, así como cuando leemos con anteojos o miramos a través de un microscopio no tomamos en cuenta las respectivas teorías ópticas (aunque en ciertos contextos podría ser imprescindible hacerlo). No es posible, en cambio, prescindir de algunos enunciados verdaderos que expresan propiedades de las partículas a las cuales intentamos referimos.

Supongamos que, al observar ciertas huellas en una fotografía, identificamos el suceso que consiste en la desintegración de un pión neutro. Al hacer esta interpretación, presuponemos que un pión neutro $\left(\pi^{\circ}\right)$ se desintegra en dos fotones $(\gamma)$, y cada uno de éstos, a su vez, produce un par electrón-positrón $\left(e^{-}, e^{+}\right)$, los cuales dejan huellas divergentes en la cámara de burbujas. Consideramos, entonces, que el enunciado $\pi^{\circ} \rightarrow \gamma+\gamma \rightarrow 2 e^{-}+2 e^{+}$es verdadero. Sin aceptar este contenido mínimo de teoría no podemos pretender, en esta situación, hacer referencia a una partícula tal como un pión neutro. Si ese enunciado fuera falso, las huellas de la fotografía podrían interpretarse de otro modo, de manera tal que revelaran la presencia de otra clase de partícula. Pretenderíamos, quizá, referirnos a un pión neutro, pero el acto de referencia 
fracasaría; y la causa de este fracaso sería, precisamente, el hecho de haber empleado una descripción falsa de esa clase de partícula.

La situación es mucho más compleja cuando se trata de identificar otros tipos de partículas. Analizaremos sólo algunos casos posibles. La situación más simple es la identificación de las partículas cargadas eléctricamente, cuando éstas son estables o tienen una larga vida media $\left(10^{-8} \mathrm{~s}\right.$ o más). Ésta es la clase de partículas que dejan huellas en una cámara de burbujas. Para identificar una partícula de este tipo, necesitamos solamente una descripción verdadera de alguna propiedad causal relevante de la partícula, tal como la de producir una huella de tales y cuales características en ciertas condiciones. En cambio, si se quiere identificar una partícula eléctricamente neutra o una partícula cargada de vida muy breve $\left(10^{-9} \mathrm{~s}\right.$ o menos, hasta $10^{-23} \mathrm{~s}$ ), no es posible observar sus huellas, ya que no las producen. La identificación de esta clase de partículas debe hacerse indirectamente, por medio de los productos de su desintegración, si son partículas inestables; o por medio de la interacción con otras partículas, cuando son partículas estables que no se desintegran. La identificación del neutrón y del neutrino nos proporcionan, respectivamente, ejemplos de ambas posibilidades.

En el conocido suceso de la desintegración beta, un neutrón se desintegra espontáneamente, en un tiempo promedio de quince minutos, produciendo un protón, un electrón y un antineutrino electrónico $\left(n \rightarrow p+e^{-}+\overline{n e}\right)$. Detectamos la ocurrencia de ese suceso al observar las huellas que dejan las partículas cargadas resultantes. Al identificar de esta manera la presencia de un neutrón, presuponemos que es verdad que se desintegra de ese modo y no de otro. Si el enunciado $n \rightarrow p+e^{-}+\overline{n e}$ fuera falso, no podríamos decir que hemos identificado un neutrón en caso de que observáramos las huellas de las partículas cargadas correspondientes. Podría tra- 
tarse de cualquier otra partícula desconocida o no. Para hacer referencia al neutrón necesitamos presuponer la verdad de este enunciado teórico.

El ejemplo de la desintegración beta es especialmente instructivo porque implica muchos otros aspectos teóricos. Ante todo, comporta la presencia de partículas estables y eléctricamente neutras, como los neutrinos, que no pueden ser detectados por sus huellas, puesto que no las dejan, ni por sus productos de desintegración, puesto que no se desintegran. La existencia del neutrino fue postulada por razones puramente teóricas ${ }^{14}$ que incluían el presupuesto de que ciertas leyes de conservación (del impulso, de la masa-energía y de determinados números cuánticos) eran verdaderas. Por consiguiente, la desintegración beta no podía ser una excepción, y la presencia del neutrino era necesaria para que las leyes de conservación no fueran violadas. Advertimos, entonces, que en la desintegración beta del neutrón también están presupuestas estas leyes de conservación. ${ }^{15}$

Las partículas neutras y estables, como los neutrinos, sólo pueden detectarse por medio de su interacción, generalmente concretada en colisiones violentas, con otras partículas cargadas o inestables. Al interpretar los resultados de esta interac-

${ }^{14}$ Cfr. Ne'eman y Kirsh, 1987, caps. 3 y 4 .

15 Actualmente la desintegración beta del neutrón se explica en el nivel de las partículas elementales de la siguiente manera. El neutrón se considera compuesto de tres quarks, uno up y dos down $(u-d-d)$, y el proton de dos up y uno down $(u-u-d)$. Cuando uno de los quarks $d$ del neutrón emite un bosón $W^{-}$, se transforma en un quark $u$, y se recombina con los otros quarks $u$ y $d$ del neutrón para formar un protón. Por su parte, el boson $W^{-}$ se desintegra enseguida en un electrón y un antineutrino electrónico. El proceso de la desintegración beta está gobernado por la interacción débil, de la cual el $W^{-}$es uno de los mediadores. El descubrimiento de los bosones vectoriales $W^{+}, W^{-}$y $Z^{\circ}$, mediadores de la interacción nuclear débil, es seguramente un caso paradigmático de identificación de partículas elementales, pero sería demasiado complejo como para analizarlo aquí. 
ción siempre se ponen en juego ciertas leyes de conservación, que establecen los límites de las interacciones posibles. Si estas leyes no estuvieran presupuestas, los resultados observados podrían interpretarse de otra manera. Así, por ejemplo, se sabe que los neutrinos y antineutrinos electrónicos pueden interactuar, aunque en una proporción muy baja, con los protones y electrones. En este caso, la interpretación de las interacciones hace intervenir a la ley de conservación del número electrónico, la cual se utiliza para discriminar entre las reacciones permitidas y las interdictas. Esta ley asigna el valor 1 al $e^{-}$ y al $n e$, y -1 al $e^{+}$y al $\overline{n e}$ y establece que la suma de los números electrónicos de las partículas debe ser la misma antes y después de la interacción. Se sigue de ello que las interacciones $n e+n \rightarrow p+e^{-}$y $\overline{n e}+p \rightarrow n+e^{+}$son posibles; mientras que $n e+p \rightarrow n+e^{+}$y $\overline{n e}+n \rightarrow p+e^{-}$no lo son, puesto que no conservan el número electrónico. Cuando interpretamos una fotografía de un detector de partículas que muestra las huellas de un protón y un electrón, apelamos a esta ley de conservación para concluir que se trata del resultado de la interacción $n e+n$. Si no empleáramos dicha ley, también podríamos haber concluido que la reacción era $\overline{n e}+n$. Al observar las huellas resultantes de $p$ y de $e^{-}$, identificamos a un neutrino electrónico y no a su antineutrino porque presuponemos que la ley de conservación del número electrónico es verdadera. De allí concluimos que la interacción observada es $n e+n \rightarrow p+e^{-}$.

El último ejemplo que analizaremos es el de la identificación de los quarks, los componentes elementales de los hadrones. Ésta es más indirecta que la de otras partículas cargadas y requiere la intervención de un mayor número de presupuestos teóricos. ${ }^{16}$ Ante todo, según la teoría del confinamiento de los quarks, éstos no pueden ser extraídos del interior de los hadrones y, por tanto, no existen como partículas libres en la natura-

Cfr. Shrader-Frechette, 1982. 
leza. Por esta razón, pese a estar dotados de una carga eléctrica característica (fraccionaria), no es posible detectar sus huellas en fotografías. Uno de los métodos de identificación de quarks consiste en producir la aniquilación de un par electrón-positrón haciéndolos colisionar a altas energías. El resultado que se observa es la producción de chorros de hadrones muy energéticos (generalmente mesones $K$ y otros) que se agrupan en direcciones opuestas. Los expertos concluyen que este fenómeno es una manifestación de los quarks y que, consiguientemente, éstos han sido observados. Sin embargo, de hecho tenemos solamente huellas de partículas de otras especies: electrones y positrones al comienzo y hadrones diversos al final del proceso. Para poder afirmar que allí se ha identificado un quark es necesario recurrir a una compleja red teórica que conecta la aniquilación de un par electrón-positrón con la producción de chorros de hadrones. En forma muy esquemática esta teoría es la siguiente. ${ }^{17} \mathrm{El}$ electrón y el positrón que chocan a gran energía se aniquilan mutuamente produciendo un fotón llamado virtual, el cual, a su vez, origina una pareja quark-antiquark. Este par conserva la elevada energía de las partículas incidentes y mediante tal energía consigue hacer pasar al estado real alguno de los infinitos pares de quark-antiquark que existen en estado virtual en el vacío. ${ }^{18}$ Puesto que los quarks no pueden quedar libres,

17 Para mayores detalles cfr. Fritzsch, 1982, pp. 185-217.

18 Probablemente sería mejor hablar de campos de quarks que llenan el espacio vacío de materia. Por comodidad empleo el lenguaje corpuscular, que se expresa en términos de partículas virtuales. La ontología de las teorías cuánticas de campos es una cuestión que permanece en la oscuridad. R. Feynman, uno de sus creadores, eligió exponer la electrodinámica cuántica (QED) en el lenguaje de una ontología de partículas. Esto tiene la desventaja de comprometerse con entidades tan extrañas como las partículas virtuales o el llamado vacío cuántico, en el cual estas partículas se crean y se aniquilan de manera constante. El comportamiento de tales entidades puede resultar verdaderamente "absurdo" (Feynman, 1985, p. 10) si se quiere acomodarlo en el marco del concepto tradicional de partícula. El punto de vista de una ontología de campos, aunque no permite la visualización de los procesos de interacción, parece más prometedor, 
cada quark se une con un antiquark (o a la inversa) para formar un mesón. Este proceso se denomina hadronización de los quarks. Los chorros de hadrones resultantes se consideran los productos de desintegración de los quarks producidos en la colisión electrón-positrón. Las leyes de conservación, como la del impulso, se utilizan para interpretar todo el proceso. La suma de los impulsos de los hadrones de cada chorro debe ser igual al impulso del quark producido en la colisión; por su parte, el impulso de este quark será igual al del respectivo electrón o positrón incidente. Con base en todos estos supuestos teóricos, es posible decir que se ha identificado un quark cuando se observa una fotografía, reconstruida por computador, de los característicos chorros de hadrones.

La identificación de las partículas elementales y subatómicas constituye un ejemplo extraído de la práctica científica real. Por medio de él podemos percatarnos de la complejidad de las hipótesis teóricas implicadas en los actos de referencia efectuados en este ámbito de la física. La referencia a las partículas no sólo no puede fundarse en la ostensión directa ni en el empleo de descripciones falsas, sino que presupone la aceptación (ciertamente sólo provisoria) de la verdad de numerosos enunciados, que van desde la simple descripción de los poderes causales de una partícula hasta las recónditas teorías de la cromodinámica cuántica.

\section{Conservación de la referencia}

Si consideramos ahora la referencia de teorías científicas en su totalidad, advertimos que la identificación de los referentes no es posible si no se conservan como verdaderos algunos enunciados. Retomemos el ejemplo de las dos teorías de partículas elementales, $T_{1}$ y $T_{2}$, que tienen en común ciertos términos como "electrón". Supongamos que $T_{1}$ ha sido rechazada, y que

por cuanto podría librarnos de algunas paradojas. No obstante, presenta sus propios problemas y se halla todavía en desarrollo (cfr. Teller, 1990). 
se ha reemplazado por $T_{2}$. ¿Cómo puede saberse que $T_{1}$ y $T_{2}$ se refieren a las mismas entidades? (en nuestro caso, a electrones). 0 más precisamente, ¿̇cómo se garantiza que $T_{2}$ conserva la referencia de $T_{1}$ ? La respuesta es que puede afirmarse que $T_{1}$ y $T_{2}$ se refieren a electrones si algunos enunciados de $T_{1}$ que emplean el término "electrón" y se admiten como verdaderos en $T_{1}$, se admiten también como verdaderos en $T_{2}$. Si todos los enunciados de $T_{1}$ acerca de electrones se consideraran como falsos en $T_{2}$, no podría decirse, entonces, que $T_{1}$ y $T_{2}$ se refieren ambas a las mismas entidades, es decir, a electrones. La conservación de la referencia implica la conservación de la verdad de ciertos enunciados en el pasaje de una teoría a otra. Así, como veremos, podemos afirmar fundadamente que la mecánica newtoniana y la mecánica relativista tienen el mismo referente, ${ }^{19}$ porque muchos enunciados que son verdaderos en la teoría clásica también se consideran verdaderos en la teoría relativista.

Podemos establecer que, en general, dos teorías tienen un mismo referente cuando: a) tienen un término común para nombrar una cierta clase de entidades (o bien, varios términos diferentes que son sinónimos); y b) algunos enunciados en los que se emplea ese término se consideran verdaderos en ambas teorías.

La necesidad de contar con enunciados verdaderos para conservar la referencia de una teoría es una consecuencia del hecho de que no hay referencia exitosa por medio de enunciados o descripciones falsas. Si la referencia en el plano del conocimiento teórico sólo es posible mediante el uso de alguna descripción verdadera, es natural que la identidad de la referencia de dos o más teorías sea garantizada únicamente cuando algunos enunciados comunes a todas las teorías se consideren como verdaderos en todas esas teorías.

19 Pasando por alto conscientemente las diversas teorías holistas acerca del cambio de significado de los términos, teorfas que hoy se hallan más bien desacreditadas. 
En este punto surge de inmediato la pregunta acerca de si, de una manera impensada, no hemos utilizado la idea de verdad absoluta al afirmar que la determinación del referente de una teoría presupone el empleo de alguna descripción verdadera, o al sostener que una teoría debe considerar como verdaderas a determinadas verdades de otra teoría para garantizar que ambas son teorías acerca de lo mismo. La respuesta es que tales descripciones o enunciados verdaderos deben ser únicamente verosímiles. Son igualmente verosímiles y revisables como cualquier otro enunciado de la teoría. Sin embargo, en el momento en que se los emplea en un acto de referencia no pueden considerarse como falsos o carentes de verosimilitud. En ese momento deben tratarse como aproximadamente verdaderos. Ello no implica que en un tiempo posterior no puedan ser falseados. Pero cuando se los rechace, considerándolos falsos, ya no podrán utilizarse en actos de referencia como los que hemos analizado. Para cumplir esa función serán necesarios otros enunciados que se tomen como verosímiles.

La mecánica clásica, en su relación con la mecánica relativista, proporciona uno de los ejemplos más claros y simples de aproximación a la verdad y de conservacion de la referencia. ${ }^{20}$ Podemos afirmar que la mecánica clásica $\left(T_{1}\right)$ y la mecánica relativista $\left(T_{2}\right)$ se refieren a las mismas cosas, porque hay muchos enunciados de $T_{1}$ que se consideran verdaderos en $T_{2}$. Esto sucede con todos los enunciados que se refieren a cuerpos que se mueven a velocidades bajas con respecto a la velocidad de la luz en el vacío. Más precisamente, se consideran bajas todas las velocidades tales que la razón $v^{2} / c^{2}$ es un número muy pequeño, que para cualquier fin práctico puede igualarse a cero. Sin embargo, en sentido estricto, estos enunciados de $T_{1}$ no son absolutamente verdaderos desde el punto de vista

20 Esto es así porque se trata de una teoría bien establecida (cfr. Rohrlich y Hardin, 1983). 
de $T_{2}$, sino sólo aproximadamente verdaderos. Son enunciados falsos, desde un punto de vista absoluto, pero aceptables para su empleo en cualquier fin práctico. En este caso, son enunciados aproximadamente verdaderos en un sentido numérico. $\mathrm{Si}$, por ejemplo, deseamos calcular el impulso de un cuerpo que se mueve a $135 \mathrm{~km} / \mathrm{s}$ respecto de un referencial dado, la fórmula clásica para el impulso $p=m \cdot v$ y la correspondiente fórmula relativista $p=m_{\circ} \cdot v / \sqrt{1-v^{2} / c^{2}}$ producirán resultados iguales hasta un orden de magnitud de $10^{-7}$. Esto significa que los números obtenidos en cada caso sólo comenzarán a diferir a partir del séptimo decimal. Ahora bien, en el mundo macroscópico $135 \mathrm{~km} / \mathrm{s}$ es una velocidad muy alta, mientras que $10^{-7}$ es un grado de precisión sumamente elevado, que rara vez se emplea (por supuesto, no ocurre lo mismo con velocidades a escala cósmica o mediciones a escala microfísica). No hay, por supuesto, un límite cuantitativo preciso para determinar cuándo un resultado de $T_{1}$ deja de ser aproximadamente verdadero para $T_{2}$. Pero hay casos claros: si un cuerpo se mueve a 10 o a $100 \mathrm{~km} / \mathrm{s}$, los resultados de $T_{1}$ serán verosímiles para $T_{2}$; en cambio, si se mueve a $2 / 3 \mathrm{c}$ o más, los resultados calculados en $T_{1}$ no serán verosímiles en $T_{2}$, pues la simple diferencia numérica será grande. En última instancia, la cuestión depende de aspectos pragmáticos. ${ }^{21} \mathrm{Si}$ en un contexto dado se necesitara una precisión de veinte decimales, $T_{1}$ no sería aceptable, incluso a muy bajas velocidades, y sus resultados no se considerarían verosímiles.

Podría pensarse que la necesidad de contar con al menos una descripción verdadera para identificar y conservar el referente de una teoría sólo se presenta en las teorías científicas que tratan acerca de entidades inobservables, como las de la física de partículas elementales. En cambio, en las teorías cuyos objetos son directamente perceptibles, se tendría siempre la posibili-

21 Cfr. Schwartz, 1990, pp. 410 y 415. 
dad de hacer referencia a tales objetos mediante actos de ostensión, con lo cual se podría prescindir de cualquier descripción verdadera del referente. Según parece, una teoría acerca del comportamiento de los elefantes no requiere de ningún enunciado verdadero para identificar a su referente, pues es posible señalarlo directamente. De este modo, una teoría $T_{2}$ podría rechazar la totalidad de los enunciados de otra teoría $T_{1}$ y, no obstante, no suscitar problemas acerca del hecho de que ambas se refieren al mismo tipo de objetos, es decir, a elefantes. Después de todo, los elefantes están allí a nuestra disposición, todavía ante nuestros ojos, para ser señalados. Frente a ellos podríamos decir: "todo lo que $T_{1}$ afirma acerca de eso es falso, lo correcto es lo que afirma $T_{2}$ ".

Esta idea tiene el encanto de la sencillez y del sentido común, pero ambos suelen ser engañosos. La identificación del referente de una teoría, aunque ésta se refiera exclusivamente a observables, nunca es tan sencillo como el (en apariencia) simple señalar de los niños. La identificación precisa de una entidad presupone siempre el empleo de una descripción verdadera, aunque sea de manera implícita y no consciente. En el conocimiento precientífico y en el del sentido común este hecho pasa fácilmente inadvertido, pues en una comunidad lingüística se dispone de un trasfondo amplio de conocimientos compartidos, que es el que nos permite identificar y reidentificar los objetos acerca de los cuales hablamos. Sin este conocimiento previo, la mera ostensión es ciega y no constituye un acto de referencia legítimo. No me extenderé aquí sobre este tema. Consideremos únicamente las dos teorías rivales $T_{1}$ y $T_{2}$ acerca del comportamiento de los elefantes. Si todos los enunciados de $T_{1}$ son considerados falsos en $T_{2}$, podemos decir que ambas teorías se refieren a los mismos objetos, esos elefantes que señalamos, sólo porque empleamos para identificarlos ciertas descripciones verdaderas que no pertenecen a $T_{1} \mathrm{ni}$ a $T_{2}$, sino al conocimiento del sentido común que poseemos acer- 
ca de los elefantes. Por esta razón podemos usar la ostension para mantener la referencia de $T_{1}$ cuando ésta es reemplazada por $T_{2}$. Si todo nuestra conocimiento de sentido común sobre los elefantes fuera incorporado a $T_{1}$ mediante enunciados explícitos, y $T_{2}$ rechazara todos esos enunciados, ya no podríamos establecer que $T_{1}$ y $T_{2}$ se refieren ambas a los mismos objetos. Los enunciados rechazados por $T_{2}$ incluirían, presumiblemente, las descripciones de la forma, tamaño y aspecto de los elefantes. Careciendo de todos estos conocimientos la referencia ostensiva se volvería extremadamente dudosa, si no imposible. No sabríamos con precisión qué entidades señalar para indicar los referentes comunes de $T_{1}$ y $T_{2}$. Es probable que no pudiéramos identificar tales entidades.

Podemos decir, finalmente, que no hay una diferencia esencial entre los procesos de identificación de entidades observables e inobservables. Los actos de referencia a elefantes y los actos de referencia a quarks y gluones presuponen ambos el empleo de al menos una descripción verdadera de estos tipos de objetos. Sin duda, existen diferencias de grado entre uno y otro caso. La referencia a quarks y gluones implica una buena porción de teoría física, mientras que la referencia a elefantes seguramente es posible mediante un número menor de enunciados y de carácter más sencillo. Por otra parte, disponemos de bastantes conocimientos de sentido común acerca de los elefantes, lo cual nos permite asegurar la referencia al hablar, aun careciendo de una teoría acerca de ellos. En cambio, tenemos muy escaso conocimiento precientífico, o quizá ninguno en absoluto, acerca de quarks y gluones, y, por esa razón, necesitamos la teoría de partículas correspondiente, o al menos una parte de ella, para poder identificar estas entidades (que, evidentemente, no forman parte de la ontología del sentido común). Si rechazáramos todos los enunciados de la teoría de los quarks, no tendríamos posibilidad de hacer referencia a esta clase de cosas. Así pues, la diferencia en el modo de 
referirse a ambas clases de entidades, elefantes y quarks, no está determinada sólo por el hecho de que unas sean directamente observables y las otras no, sino también por los distintos tipos de conocimiento que se requieren para la identificación de cada una. ${ }^{22}$

\section{BIBLIOGRAFIA}

Boyd, R., 1989, "What Realism Implies and What it Does Not", Dialectica, no. 43, pp. 5-29.

1990, "Realism, Approximate Truth, and Philosophical Method", en C. Wade Savage (comp.), Scientific Theories, University of Minnesota Press, Minneapolis.

Brush, S.G., 1980, "The Chimerical Cat: Philosophy of Quantum Mechanics in Historical Perspective", Social Studies of Science, no. 10 , pp. 393-447.

Cohen-Tannoudji, G. y M. Spiro, 1990, La matière-espace-temps, Gallimard, París.

Deligeorges, S. (comp.), 1984, Le monde quantique, Editions du Seuil, París.

D'Espagnat, B., 1981, A la recherche du réel. Le regard d'un physicien, Bordas, París.

Devitt, M., 1984, Realism and Truth, Basil Blackwell, Oxford.

Dummett, M., 1982, “Realism”, Synthese, no. 52, pp. 55-112.

Feynman, R.P., 1985, QED: The Strange Theory of Light and Matter, Princeton University Press, Princeton.

Fine, A., 1986, "Unnatural Attitudes: Realist and Instrumentalist Attachments to Science", Mind, no. 95, pp. 149-179.

Fritzsch, H., 1982, Los quarks, la materia prima de nuestro Universo, Alianza, Madrid.

Gibbins, P., 1987, Particles and Paradoxes. The Limits of Quantum Logic, Cambridge University Press, Cambridge.

${ }^{22}$ Mi agradecimiento a la Fundación Antorchas, la cual hizo posible que pudiera continuar la investigación sobre este tema. 
Grayling, A.C., 1992, "Epistemology and Realism", Proceedings of the Aristotelian Society, no. 92, pp. 47-65.

Harre, R., 1986, Varieties of Realism. A Rationale for the Natural Sciences, Basil Blackwell, Oxford.

Healey, R.A., 1991, "Holism and Nonseparability", Journal of Philosophy, no. 88, pp. 393-421.

Hellman, G., 1983, "Realist Principles", Philosophy of Science, no. 50, pp. 227-249.

Hooker, C.A., 1987, "A Naturalist Realism", Revue Internationale de Philosophie, no. 160, pp. 5-28.

Horwich, P., 1982, "Three Forms of Realism", Synthèse, no. 51, pp. 181-201.

Jones, R., 1991, "Realism About What?", Philosophy of Science, no. 58, pp. 185-202.

Laudan, L., 1984, Science and Values, University of California Press, Berkeley.

Mermin, D., 1981, "Quantum Mysteries for Anyone", Journal of Philosophy, no. 78, pp. 397-408.

Ne'eman, Y. y Y. Kirsh, 1987, The Particle Hunters, Cambridge University Press, Cambridge.

Newton-Smith, W.H., 1981, The Rationality of Science, Routledge and Kegan Paul, Boston.

-, 1989, "The Truth in Realism", Dialectica, no. 43, pp. 31-45.

Ortoli, S. y J.P. Pharabod, 1984, Le cantique des quantiques, Editions La Découverte, París.

Rae, A.I.M., 1986, Quantum Physics: Illusion or Reality?, Cambridge University Press, Cambridge.

Redhead, M., 1989, "The Nature of Reality", British Journal for the Philosophy of Science, no. 40, pp. 429-441.

Rohrlich, F. y L. Hardin, 1983, "Established Theories", Philosophy of Science, no. 50, pp. 603-617.

Schwartz, R.J., 1990, “Approximate Truth, Idealization and Ontology", Southern Journal of Philosophy, no. 28, pp. 409-425.

Selleri, F., 1986, El debate de la teoría cuántica, Alianza, Madrid.

Shrader-Frechette, K.S., 1982, "Quark Quantum Number and the Problem of Microphysical Observation", Synthèse, no. 50, pp. 125-145.

Taylor, B., 1987, "The Truth in Realism", Revue Internationale de Philosophie, no. 160, pp. 45-63. 
Teller, P., 1979, "Quantum Mechanics and the Nature of Continuous Physical Quantities", Journal of Philosophy, no. 76, pp. 345-361. _, 1986, "Relational Holism in Quantum Mechanics", British Journal for the Philosophy of Science, no, 37, pp. 71-81.

1990, "Prolegomen to a Proper Interpretation of Quantum Field Theory", Philosophy of Science, no. 57, pp. 594-618.

Van Fraassen, B.C., 1980, The Scientific Image, Clarendon Press, Oxford.

Recibido: 14 de mayo de 1992 


\section{SUMMARY}

This paper attempts to determine the commitements of the realist view of scientific theories. First, it distinguishes between ontological realism and epistemological realism. Then, it affirms that the latter is committed with the concepts of reference and truthlikeness. It rejects the so-called referential realism, and against this, it maintains that it is not possible to perform genuine acts of reference by means of false descriptions. Every act of reference needs a true description. It exemplifies this thesis with the problem of identifying elementary particles. Finally, it concludes by analysing the conditions which are necessary and sufficient for the conservation of the reference when a change of theory takes place. 